\title{
A Binary Model for the UV-upturn of Elliptical Galaxies
}

\author{
Z. Han ${ }^{1}$, Ph. Podsiadlowski ${ }^{2}$, A.E. Lynas-Gray ${ }^{2}$ and K. Schawinski ${ }^{2}$ \\ ${ }^{1}$ National Astronomical Observatories / Yunnan Observatory, \\ the Chinese Academy of Sciences, Kunming 650011, China \\ email: zhanwenhan@hotmail.com \\ ${ }^{2}$ University of Oxford, Department of Astrophysics, Keble Road, Oxford OX1 3RH
}

\begin{abstract}
The discovery of an excess of light in the far-ultraviolet (UV) in 1969 in elliptical galaxies was a major surprise. While it is now clear that this UV excess (UV-upturn) is probably caused by an old population of helium-burning stars. Han et al. $(2002,2003)$ proposed a binary model for the formation of hot subdwarfs (helium burning stars) and the model can reproduce the observations in our Galaxy. By applying the binary model to the study of evolutionary population synthesis, we have obtained an a priori model for the UV-upturn of elliptical galaxies. The model shows that the UV-upturn is most likely resulted from binary interactions and it is universal (not very much metallicity-dependant) in ellipticals. This has major implications for understanding the evolution of the UV-upturn and elliptical galaxies in general; contrary to previous postulates, it implies that the UV-upturn is not a sign of age, but could be a potentially powerful indicator for a recent minor burst of star-forming activity.
\end{abstract}

Keywords. ultraviolet: galaxies; galaxies: elliptical and lenticular, cD; stars: binaries: close

A long-standing problem in the study of elliptical galaxies is the far-ultraviolet (UV) excess (or UV-upturn) in their spectra. It is now clear that UV-upturn is caused by an old population of helium-burning stars or their descendants with a characteristic surface temperature of 25,000 K (Ferguson et al. 1991), also known as hot subdwarfs. The origin for the population of these hot, blue stars in an otherwise red population has, however, remained a major mystery. Two scenarios, referred to as the high- and the lowmetallicity scenario, have been advanced (Yi, Demarque \& Kim 1997; Lee 1994), though these scenarios are quite ad hoc. Both of these scenarios ignore the effects of binary evolution. On the other hand, hot subdwarfs have long been studied in our own Galaxy, and it is now well established that the vast majority of (and quite possibly all) Galactic hot subdwarfs are the results of binary interactions, where a star loses all of its envelope near the tip of the red-giant branch by mass transfer to a companion star or ejection in a common-envelope phase, or where two helium white dwarfs merge with a combined mass larger than $\sim 0.35 M_{\odot}$ (see Han et al. 2002, 2003 for references and details). In all of these cases, the remnant star ignites helium and becomes a hot subdwarf. The key feature of these binary channels is that they provide the missing physical mechanism for ejecting the envelope and producing a hot subdwarf. Moreover, since it is known that these hot subdwarfs provide an important source of far-UV light in our own Galaxy, it is reasonable to assume that they also contribute to the far-UV in elliptical galaxies.

To model the effects of binary evolution on the spectral appearance of elliptical galaxies, we have performed population synthesis study of galaxies that includes binary evolution. It is based on a binary population synthesis model (Han et al. 2002; Han et al. 2003) that has been calibrated to reproduce the short-period hot subdwarf binaries in our own Galaxy that make up the majority of Galactic hot subdwarfs. The population synthesis 

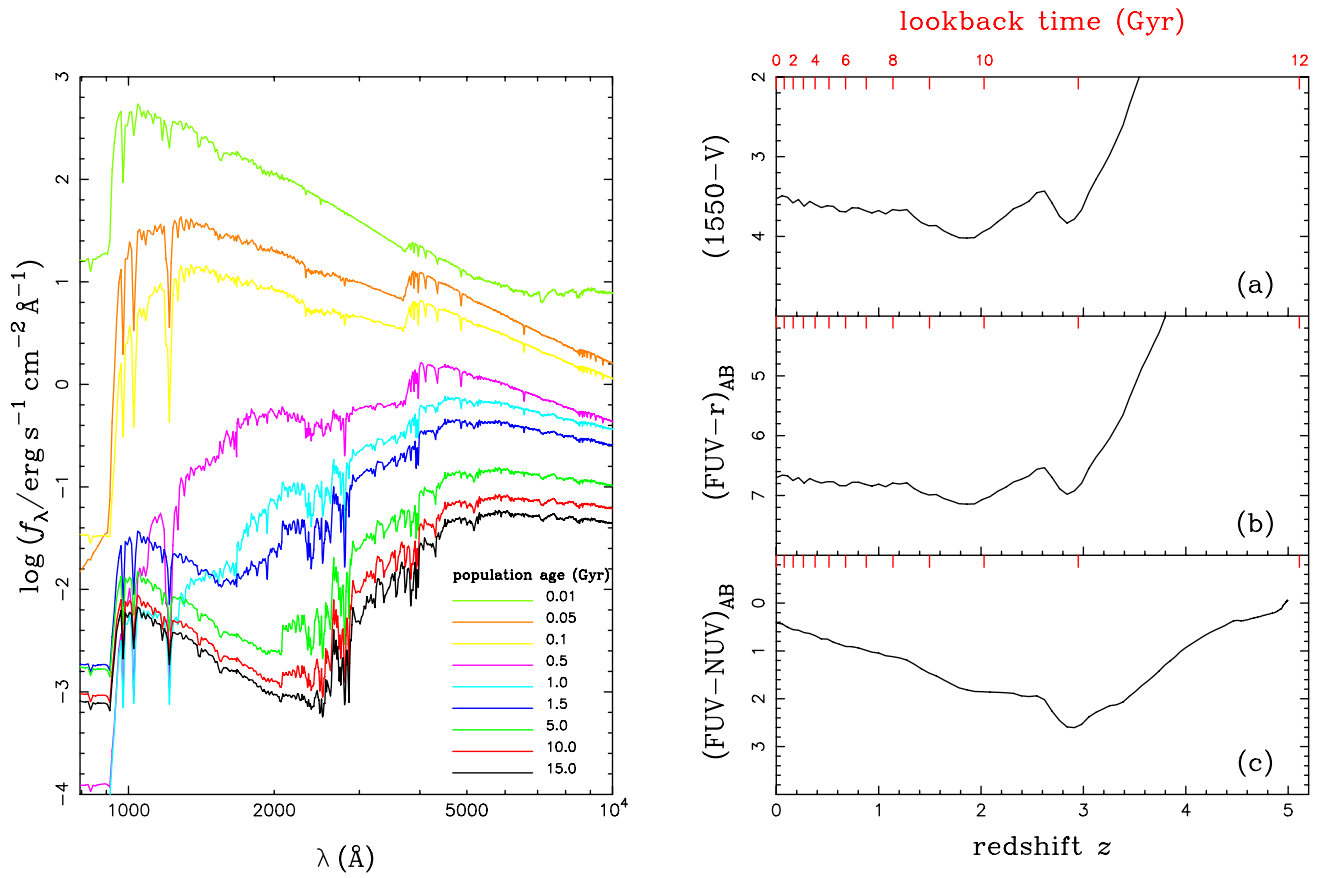

Figure 1: Left panel: the evolution of SED (restframe and intrinsic) for a simple stellar population (including binaries) of $10^{10} M_{\odot}$ at a distance of $10 \mathrm{pc}$. We see that hot subdwarfs originating from binary interactions dominate the FUV after $\sim 1$ Gyr. Right panel: The evolution of UV-upturn (restframe and intrinsic) with redshift (lookback time) for a simple stellar population (including binaries). FUV, NUV are GALEX colours, $\mathrm{r}$ is a SDSS colour, $\mathrm{AB}$ means in $\mathrm{AB}$ magnitude. We adopted a star-formation redshift of $z_{\mathrm{f}}=5$, and cosmological parameters of $H_{0}=72 \mathrm{~km} / \mathrm{s} / \mathrm{Mpc}, \Omega_{\mathrm{M}}=0.3$ and $\Omega_{\Lambda}=0.7$.

model follows the detailed time evolution of both single and binary stars, including all binary interactions, and is capable of simulating galaxies of arbitrary complexity, provided the star-formation history is specified.

To obtain galaxy colours and spectra, we have calculated spectra for hot subdwarfs using plane-parallel static model stellar atmospheres computed with the ATLAs9 (Kurucz 1992) stellar atmosphere code. For the spectrum and colours of other single stars, we use the comprehensive BaSeL library of theoretical stellar spectra (Lejeune, Cuisinier \& Buser 1997; Lejune, Cuisinier \& Buser 1998).

This work was in part supported by the NSFC under Grant Nos. 10433030 and 10521001, the CAS under Grant No. KJCX2-SW-T06 (Z.H.)

\section{References}

Ferguson, H.C. et al., 1991, ApJ, 382, L69

Han, Z., Podsiadlowski, Ph., Maxted, P.F.L., Marsh, T.R., \& Ivanova, N., 2002, MNRAS, 336, 449

Han, Z., Podsiadlowski, Ph., Maxted, P.F.L., \& Marsh, T.R., 2003, MNRAS, 341, 669

Kurucz, R.L., 1992, in Barbuy B., \& Renzini A., eds, Proc. IAU Symp. 149, The Stellar Population of Galaxies, Kluwer, Dordrecht, p.225

Lee, Y.W., 1994, ApJ, 430, L113

Lejeune, T., Cuisinier, F., \& Buser, R., 1997, A\&AS, 125, 229

Lejeune, T., Cuisinier, F., \& Buser, R., 1998, A\&AS, 130, 65

Yi, S.K., Demarque, P., \& Kim, Y.C., 1997, ApJ, 482, 677 